\title{
Inequalities for the truncated Hilbert transform and the segment multiplier
}

\author{
Adam Osȩkowski
}

Received: 18 November 2012 / Accepted: 13 May 2013 / Published online: 31 May 2013

(C) The Author(s) 2013. This article is published with open access at Springerlink.com

\begin{abstract}
The paper is devoted to the study of $\log L$ inequalities and other related bounds for two classical operators on the real line: the truncated Hilbert transform and the segment multiplier. Using duality, these estimates are deduced from corresponding sharp exponentialtype bounds, the proofs of which rest on the construction of appropriate harmonic functions on the strip $[-1,1] \times \mathbb{R}$ and transference-type arguments.
\end{abstract}

Keywords Hilbert transform · Truncated Hilbert transform - Segment multiplier · LlogL inequality $\cdot$ Best constants

Mathematics Subject Classification (2000) $\quad 42 \mathrm{~B} 10 \cdot 31 \mathrm{H} 05$

\section{Introduction}

Our motivation comes from the question concerning logarithmic estimates for some classical Fourier multipliers on the real line. However, to introduce the background and indicate the connections with other celebrated results from the literature, we start with the periodic setting. Suppose that $f(\zeta)=\sum_{n \in \mathbb{Z}} \hat{f}(n) \zeta^{n}$ is a complex-valued integrable function defined on the unit circle $\mathbb{T}=\{\zeta \in \mathbb{C}:|\zeta|=1\}$. Here the symbol $\hat{f}(n)=\frac{1}{2 \pi} \int_{-\pi}^{\pi} f\left(e^{i \theta}\right) e^{-i n \theta} d \theta$ denotes the $n$-th Fourier coefficient of $f$. For a given $p \geq 1$, let $H^{p}(\mathbb{T}, \mathbb{C})$, the Hardy space, consist of all $f \in L^{p}(\mathbb{T}, \mathbb{C})$ satisfying $\hat{f}(n)=0$ for $n<0$. Then $H^{p}(\mathbb{T}, \mathbb{C})$ is a closed subspace of $L^{p}(\mathbb{T}, \mathbb{C})$ and can be identified with the class of analytic functions on the unit disc $\mathbb{D}$. The Riesz projection (or analytic projection) $P_{+}^{\mathbb{T}}: L^{p}(\mathbb{T}, \mathbb{C}) \rightarrow H^{p}(\mathbb{T}, \mathbb{C}$ ), is the operator given by the formula

\footnotetext{
A. Osȩkowski ( $\varangle)$

Faculty of Mathematics, Informatics and Mechanics, University of Warsaw, Banacha 2,

02-097 Warsaw, Poland

e-mail: ados@mimuw.edu.pl
} 


$$
P_{+}^{\mathbb{T}} f(\zeta)=f_{+}(\zeta)=\sum_{n \geq 0} \hat{f}(n) \zeta^{n}, \quad \zeta \in \mathbb{T}
$$

The complementary operator $P_{-}^{\mathbb{T}}=I-P_{+}^{\mathbb{T}}$ is called the co-analytic projection. These two operators are strictly connected to another classical object, the Hilbert transform (conjugate function) on the unit circle, which is defined by

$$
\mathcal{H}^{\mathbb{T}} f(\zeta)=-i \sum_{n \in \mathbb{Z}} \operatorname{sgn}(n) \hat{f}(n) \zeta^{n}, \quad \zeta \in \mathbb{T}
$$

Here $\operatorname{sgn}(n)=n /|n|$ for $n \neq 0$ and $\operatorname{sgn}(0)=0$. An alternative definition of $\mathcal{H}^{\mathbb{T}}$ is by the use of the singular integral

$$
\mathcal{H}^{\mathbb{T}} f\left(e^{i \theta}\right)=\frac{1}{2 \pi} \mathrm{p} \cdot \mathrm{v} \cdot \int_{-\pi}^{\pi} f\left(e^{i t}\right) \cot \frac{\theta-t}{2} \mathrm{~d} t .
$$

A fundamental result of Riesz [19] asserts that the operator $P_{+}^{\mathbb{T}}$ (equivalently, the Hilbert transform $\left.\mathcal{H}^{\mathbb{T}}\right)$ is bounded on $L^{p}(\mathbb{T}, \mathbb{C})$ for $1<p<\infty$. The question about the precise value of the norms of these operators has gathered a lot of interest in the literature. Gohberg and Krupnik [9] determined the norm of the Hilbert transform for $p=2^{k}, k=1,2, \ldots$. Namely, we have

$$
\left\|\mathcal{H}^{\mathbb{T}}\right\|_{L^{p}(\mathbb{T}, \mathbb{C}) \rightarrow L^{p}(\mathbb{T}, \mathbb{C})}=\cot (\pi /(2 p)) .
$$

For the remaining $p$ 's, the norms of $\mathcal{H}^{\mathbb{T}}$ acting on real $L^{p}$ spaces were found by Pichorides [18] and, independently, by Cole (unpublished; consult Gamelin [8]):

$$
\left\|\mathcal{H}^{\mathbb{T}}\right\|_{L^{p}(\mathbb{T}, \mathbb{R}) \rightarrow L^{p}(\mathbb{T}, \mathbb{R})}=\cot \left(\pi /\left(2 p^{*}\right)\right),
$$

where $p^{*}=\max \{p, p /(p-1)\}$. See also Essén [5] and Verbitsky [21]. These norms do not change while passing to the complex $L^{p}$ spaces (see e.g. Pełczyński [17]):

$$
\left\|\mathcal{H}^{\mathbb{T}}\right\|_{L^{p}(\mathbb{T}, \mathbb{C}) \rightarrow L^{p}(\mathbb{T}, \mathbb{C})}=\cot \left(\pi /\left(2 p^{*}\right)\right), \quad 1<p<\infty .
$$

For the Riesz projection, Hollenbeck and Verbitsky [11] (see also [12]) proved that

$$
\left\|P_{ \pm}^{\mathbb{T}}\right\|_{L^{p}(\mathbb{T}, \mathbb{C}) \rightarrow L^{p}(\mathbb{T}, \mathbb{C})}=\csc (\pi / p), \quad 1<p<\infty .
$$

The above facts have their non-periodic counterparts. For a given $f: \mathbb{R} \rightarrow \mathbb{C}$, let

$$
\hat{f}(\xi)=\int_{\mathbb{R}} f(x) e^{-2 \pi i x \xi} \mathrm{d} x, \quad \xi \in \mathbb{R},
$$

denote the Fourier transform of $f$. Then the non-periodic analytic and co-analytic projections $P_{+}^{\mathbb{R}}, P_{-}^{\mathbb{R}}$, and Hilbert transform $\mathcal{H}^{\mathbb{R}}$ on the line are given by

$$
P_{+}^{\mathbb{R}} f(x)=\int_{\mathbb{R}} \hat{f}(\xi) \chi_{[0, \infty)}(\xi) e^{2 \pi i x \xi} \mathrm{d} \xi, \quad P_{-}^{\mathbb{R}} f(x)=\int_{\mathbb{R}} \hat{f}(\xi) \chi_{(-\infty, 0]}(\xi) e^{2 \pi i x \xi} \mathrm{d} \xi
$$

and

$$
\mathcal{H}^{\mathbb{R}} f(x)=-i \int_{\mathbb{R}} \hat{f}(\xi)\left(-\chi_{(-\infty, 0]}+\chi_{[0, \infty)}(\xi)\right) e^{2 \pi i x \xi} \mathrm{d} \xi
$$


Using the standard argument known as "blowing up the circle", which is due to Zygmund ([22], Chapter XVI, Theorem 3.8), it can be shown that the corresponding $L^{p}$ norms of these operators are the same as in the periodic setting.

We will be interested in related Fourier multipliers on the line: the so-called truncated Hilbert transform $\mathcal{H}_{r}$ and the segment multiplier $\mathcal{S}_{[a, b]}$; here $r$ is an arbitrary positive number and $[a, b] \subset \mathbb{R}$ is a given subinterval. The formal definition of these operators is as follows: for any function $f$ on $\mathbb{R}$ and any $x \in \mathbb{R}$,

$$
\mathcal{H}_{r} f(x)=-i \int_{\mathbb{R}}\left(-\chi_{(-\infty,-r)}(\xi)+\chi_{(r, \infty)}(\xi)\right) \hat{f}(\xi) e^{2 \pi i x \xi} \mathrm{d} \xi
$$

and

$$
\mathcal{S}_{[a, b]} f(x)=\int_{\mathbb{R}} \hat{f}(\xi) \chi[a, b] e^{2 \pi i x \xi} \mathrm{d} \xi .
$$

The action of these operators on $L^{p}$ spaces were studied by De Carli and Laeng in [3] and [4]. One can find there the proofs of the identities

$$
\left\|\mathcal{H}_{r}\right\|_{L^{p}(\mathbb{T}, \mathbb{R}) \rightarrow L^{p}(\mathbb{T}, \mathbb{R})}=\left\|\mathcal{S}_{[a, b]}\right\|_{L^{p}(\mathbb{T}, \mathbb{R}) \rightarrow L^{p}(\mathbb{T}, \mathbb{R})}=\cot \left(\pi /\left(2 p^{*}\right)\right)
$$

for $1<p<\infty$; moreover, it was shown that the norms are the same on complex $L^{p}$ spaces. We will be interested in obtaining an appropriate version of this result for $p=1$. Neither of $\mathcal{H}_{r}$ and $\mathcal{S}_{[a, b]}$ is bounded on $L^{1}(\mathcal{T}, \mathbb{R})$, but, as usual, we can inspect the corresponding localized L $\log L$ estimate (cf. [10]). Let us formulate this bound in a more general context. Suppose that $\Psi:[0, \infty) \rightarrow[0, \infty)$ is a given nondecreasing convex function (for example, $\Psi(x)=K x \log ^{+} x$ or $\Psi(x)=K((x+1) \log (x+1)-x)$, where $K>0$ is a fixed number). There is a question about a constant $L=L_{\Psi}$ depending only on $\Psi$ such that for any Borel function $f: \mathbb{R} \rightarrow \mathbb{C}$ and any set $A \subset \mathbb{R}$,

$$
\int_{A}\left|\mathcal{H}_{r} f(x)\right| \mathrm{d} x \leq \int_{\mathbb{R}} \Psi(|f(x)|) \mathrm{d} x+L_{\Psi} \cdot|A| .
$$

Analogous problem can be posed for $\mathcal{S}_{[a, b]}$. As with any inequality of this type, the following two questions can be asked:

$1^{\circ}$ For which $\Psi$ there is an absolute finite $L_{\Psi}$ such that (3) holds?

$2^{\circ}$ For $\Psi$ as in $1^{\circ}$, what is the optimal (least possible) value of $L_{\Psi}$ ?

We study these questions in a much wider, vector-valued case. Consider the Hilbert space $\ell_{\mathbb{C}}^{2}$ with norm $|\cdot|$ and scalar product $\langle\cdot, \cdot\rangle$. Then $\mathcal{H}_{r}$ and $\mathcal{S}_{[a, b]}$ can be extended to the operators acting on $\ell_{\mathbb{C}}^{2}$-valued functions. Indeed, we may define them coordinatewise, or simply note that the previous definitions make sense in this new setting.

Throughout, $\mathcal{C}$ will be the class of all convex and strictly increasing functions $\Phi$ : $[0, \infty) \rightarrow[0, \infty)$ such that

(i) $\Phi$ is continuously differentiable on $(0, \infty)$,

(ii) $\Phi^{\prime}$ is convex on $(0, \infty)$,

(iii) $\Phi(0)=\Phi^{\prime}(0+)=0$.

Examples: $\Phi(t)=t^{p}$ for $p \geq 2 ; \Phi(t)=e^{t}-1-t$. Next, $\mathcal{C}^{*}$ will stand for the dual of $\mathcal{C}$ in the sense of Cramer transform. That is, $\Psi:[0, \infty) \rightarrow[0, \infty)$ belongs to $\mathcal{C}^{*}$ if and only if there is $\Phi \in \mathcal{C}$ such that

$$
\Psi(s)=\Phi^{*}(s):=\sup \{t s-\Phi(t): t \geq 0\} \text { for } s \geq 0 .
$$


The main results of the paper can be stated as follows.

Theorem 1.1 Let $r>0$ be fixed and $\Psi=\Phi^{*}$ be a given element of $\mathcal{C}^{*}$. Then for any Borel function $f: \mathbb{R} \rightarrow \ell_{\mathbb{C}}^{2}$ and any Borel subset $A$ of $\mathbb{R}$ we have

$$
\int_{A}\left|\mathcal{H}_{r} f(x)\right| \mathrm{d} x \leq \int_{\mathbb{R}} \Psi(|f(x)|) \mathrm{d} x+C(\Phi) \cdot|A|,
$$

where

$$
C(\Phi)=\frac{1}{\pi} \int_{-\infty}^{\infty} \frac{\Phi\left(\left|\frac{2}{\pi} \log \right| r||\right)}{r^{2}+1} \mathrm{~d} r .
$$

For any $r$ and $\Psi$, the constant $C(\Phi)$ is the best possible.

Theorem 1.2 Let $[a, b] \subset \mathbb{R}$ and $\Psi=\Phi^{*}$ be a given element of $\mathcal{C}^{*}$. Then for any Borel function $f: \mathbb{R} \rightarrow \ell_{\mathbb{C}}^{2}$ and any Borel subset $A$ of $\mathbb{R}$ we have

$$
\int_{A}\left|\mathcal{S}_{[a, b]} f(x)\right| \mathrm{d} x \leq \int_{\mathbb{R}} \Psi(|f(x)|) \mathrm{d} x+C(\Phi) \cdot|A|,
$$

where $C(\Phi)$ is given by (5).

A few remarks are in order. First, we do not know whether the constant $C(\Phi)$ in (6) is the best possible, but we will present some lower bounds for this constant in Sect. 4 below. Next, straightforward limiting arguments (put $b=0$ and let $a \rightarrow-\infty$, or put $a=0$ and let $b \rightarrow \infty$, and use Fatou's lemma) imply that (6) holds for the operators $P_{ \pm}^{\mathbb{R}}$ as well. The final remark concerns some exemplary choices for $\Psi$ :

(i) We start with the natural choice $\Psi(t)=t \log ^{+} t$. Unfortunately, this function is not in $\mathcal{C}^{*}$; in fact, neither of the inequalities (4), (6) holds with some finite $C$. Indeed, otherwise $\mathcal{H}_{r}$ or $\mathcal{S}_{[a, b]}$ would send functions bounded by 1 to bounded functions, which is false (because it is not true for $\mathcal{H}^{\mathbb{R}}$ and $P_{ \pm}^{\mathbb{R}}$ ).

(ii) One of the right choices for the $\operatorname{Llog} \mathrm{L}$ functions is given by the formula $\Psi(x)=$ $K((x+1) \log (x+1)-x)$ for a fixed $K>0$. Then $\Psi=\Phi^{*}$, where $\Phi \in \mathcal{C}$ is defined by

$$
\Phi(t)=K\left(e^{t / K}-1-t / K\right), \quad t \geq 0,
$$

and, directly from (5), we see that $C(\Phi)<\infty$ if and only if $K>2 / \pi$.

(iii) There is a different choice for a $\operatorname{LlogL}$ function. If we pick $K>0$ and put $\Phi(t)=$ $K(\cosh (t / K)-1)$, then some straightforward computations give

$$
\Psi(t)=\Phi^{*}(t)=K\left[t \operatorname{arcsinh} t-\sqrt{t^{2}+1}+1\right]
$$

and, as previously, $C(\Phi)<\infty$ if and only if $K>2 / \pi$.

(iv) Lastly, we mention here another application of the above results. Let $\Psi(t)=t^{p}$ be a power function, $1<p \leq 2$. Then $\Psi=\Phi^{*}$, where $\Phi(t)=t^{q}, 2 \leq q<\infty$, and the bounds (4), (6) lead to weak-type estimates for $\mathcal{H}_{r}$ and $\mathcal{S}_{[a, b]}$. To see this, apply (4) to $c f$ (where $c$ is a given positive parameter) and optimize over $c$ to get

$$
\sup _{A}\left\{\frac{1}{|A|^{1-1 / p}} \int_{A}\left|\mathcal{H}_{r} f(x)\right| \mathrm{d} x\right\} \leq \frac{p}{(p-1)^{1-1 / p}} C(\Phi)^{1-1 / p}\|f\|_{p} .
$$


It remains to note that the left-hand side defines a norm on the space $L^{p, \infty}$ (cf. [10]).

We would like to point out here that various versions of (4) and (6) concerning Hilbert transform and (co-)analytic projection, as well as other related operators, have been studied in depth in the literature. We refer the interested reader to the papers by Bennett [1], Essén, Shea and Stanton [6,7], Laeng [13], O'Neil and Weiss [14], the author [15,16], Pichorides [18], Zygmund [22] and references therein.

A few words about our approach and the organization of the paper. The key object is a family of certain special harmonic functions on the strip $[-1,1] \times \mathbb{R}$. These functions enable us to establish a novel $\Phi$-estimate for the Hilbert transform when restricted to bounded functions. This inequality is proved in Sect. 2 . In Sect. 3 we combine this bound with some duality arguments to deduce the estimates of Theorems 1.1 and 1.2. The final part of the paper is devoted to the sharpness: we provide lower bounds for the constants $C(\Phi)$ in (4) and (6).

\section{Sharp $\Phi$-estimates for the Hilbert transform}

We start this section with a well-known fact from complex analysis (see e.g. Theorem 4.13 in [20]), which will be needed in our further considerations. For $z=\left(z_{1}, z_{2}, \ldots\right) \in \ell_{\mathbb{C}}^{2}$, we define the conjugation by $\bar{z}=\left(\overline{z_{1}}, \overline{z_{2}}, \ldots\right)$; then, for any $w, z \in \ell_{\mathbb{C}}^{2}$, we have $\langle w, \bar{z}\rangle=\sum_{j=1}^{\infty} w_{j} z_{j}$.

Theorem 2.1 Suppose that $D$ is a given subdomain of $\mathbb{C}$ and let $D^{\prime}=\left\{(w, z) \in \ell_{\mathbb{C}}^{2} \times \ell_{\mathbb{C}}^{2}\right.$ : $\langle w, \bar{z}\rangle \in D\}$. If $\phi: D \rightarrow \mathbb{R}$ is harmonic, then $U: D^{\prime} \rightarrow \mathbb{R}$ given by $U(w, z)=\phi(\langle w, \bar{z}\rangle)$ is pluriharmonic.

It will be convenient to work with the following family of special functions. For any $t \geq 0$ let $\Phi^{(t)}: \mathbb{R}_{+} \rightarrow \mathbb{R}_{+}$be given by $\Phi^{(t)}(s)=(\max \{s-t, 0\})^{2}$. It is easy to see that these are precisely the extremal elements of the class $\mathcal{C}$ (see the identity (19) below).

Now we turn to the introduction of the main object in this paper, a certain class $\left\{V^{(t)}\right\}_{t \geq 0}$ of special harmonic functions. Let $H=\{(x, y): y>0\}$ denote the upper half-space and let $S=\left\{(x, y) \in \mathbb{R}^{2}:|x|<1\right\}$ stand for the vertical strip in $\mathbb{R}^{2}$. Fix $t \geq 0$ and define $\mathcal{V}^{(t)}: H \rightarrow \mathbb{R}$ by the formula

$$
\mathcal{V}^{(t)}(\alpha, \beta)=\frac{1}{\pi} \int_{-\infty}^{\infty} \frac{\beta \Phi^{(t)}\left(\left|\frac{2}{\pi} \log \right| r||\right)}{(\alpha-r)^{2}+\beta^{2}} \mathrm{~d} r-C\left(\Phi^{(t)}\right) .
$$

The constant $C\left(\Phi^{(t)}\right)$ [see (5)] guarantees that $\mathcal{V}^{(t)}(0,1)=0$. As a Poisson integral, $\mathcal{V}^{(t)}$ is a harmonic function on $H$; furthermore, it has the following behavior at the lower boundary of the halfplane:

$$
\lim _{(\alpha, \beta) \rightarrow(r, 0)} \mathcal{V}^{(t)}(\alpha, \beta)=\Phi^{(t)}\left(\left|\frac{2}{\pi} \log \right| r||\right)-C\left(\Phi^{(t)}\right) .
$$

Consider a conformal map $\phi(z)=i \exp (-i \pi z / 2)$, or, in real coordinates,

$$
\phi(x, y)=\left(e^{\pi y / 2} \sin (\pi x / 2), e^{\pi y / 2} \cos (\pi x / 2)\right) .
$$

We easily check that this function maps $S$ onto $H$. Finally, introduce $V^{(t)}: \bar{S} \rightarrow \mathbb{R}$ by

$$
V^{(t)}(x, y)= \begin{cases}\Phi^{(t)}(|y|)-C\left(\Phi^{(t)}\right) & \text { if }|x|=1, \\ \mathcal{V}^{(t)}(\phi(x, y)) & \text { if }|x|<1 .\end{cases}
$$


It is not difficult to verify that for $(x, y) \in S$,

$$
V^{(t)}(x, y)=\frac{1}{\pi} \int_{-\infty}^{\infty} \frac{\cos \left(\frac{\pi}{2} x\right) \Phi^{(t)}\left(\frac{2}{\pi} \log |s|+y\right)}{s^{2}-2 s \sin \left(\frac{\pi}{2} x\right)+1} \mathrm{~d} s-C\left(\Phi^{(t)}\right) .
$$

The function $V^{(t)}$ is harmonic on $S$, as a composition of a harmonic function with a conformal mapping. Therefore, it can be expressed as a real part of a certain holomorphic function $G^{(t)}$ :

$$
V^{(t)}=\operatorname{Re} G^{(t)} .
$$

Moreover, by (8), $V^{(t)}$ is a continuous function on the closure of $S$. It satisfies the symmetry condition

$$
V^{(t)}(x, y)=V^{(t)}(x,-y)=V^{(t)}(-x, y) \text { for all }(x, y) \in S .
$$

Indeed, this can be rewritten in the equivalent form

$$
\mathcal{V}^{(t)}(\alpha, \beta)=\mathcal{V}^{(t)}(-\alpha, \beta)=\mathcal{V}^{(t)}\left(\frac{\alpha}{\alpha^{2}+\beta^{2}}, \frac{\beta}{\alpha^{2}+\beta^{2}}\right),
$$

which can be verified by substitution $r:=-r$ and $r:=1 / r$ in (7).

In the lemma below, we study further properties of $V^{(t)}$, to be needed later.

Lemma 2.2 (i) We have $V^{(t)}(x, 0) \leq 0$ for all $x \in[-1,1]$.

(ii) If $x \in(-1,1)$ and $y \geq 0$, then $V_{y y y}^{(t)}(x, y) \geq 0$.

(iii) If $x \in[0,1)$ and $y \geq 0$, then $y V_{x}^{(t)}(x, y)+x V_{y}^{(t)}(x, y) \leq 0$.

(iv) For each $t \geq 0$ there are $a_{0}, a_{1}, a_{2}, \ldots \in \mathbb{C}$ such that the holomorphic function $G^{(t)}$ given by (10) satisfies $G^{(t)}(z)=\sum_{n=0}^{\infty} a_{n} z^{2 n}$ for all $z \in S$.

Proof (i) Since $\Phi^{(t)}$ is convex, (9) implies that for a fixed $x \in[-1,1]$, the function $V^{(t)}(x, \cdot)$ is also convex. Hence, by the harmonicity of $V^{(t)}$, we have $V_{x x}^{(t)} \leq 0$ on $S$ and it remains to apply (11) to get $V^{(t)}(x, 0) \leq V^{(t)}(0,0)=\mathcal{V}^{(t)}(0,1)=0$.

(ii) We have

$$
V_{y}^{(t)}(x, y)=\frac{p}{\pi} \int_{-\infty}^{\infty} \frac{\cos \left(\frac{\pi}{2} x\right)\left(\Phi^{(t)}\right)^{\prime}\left(\left|\frac{2}{\pi} \log \right| s|+y|\right) \operatorname{sgn}\left(\frac{2}{\pi} \log |s|+y\right)}{\left(s-\sin \left(\frac{\pi}{2} x\right)\right)^{2}+\cos ^{2}\left(\frac{\pi}{2} x\right)} \mathrm{d} s .
$$

Therefore, for $\varepsilon \in(0, y)$ we have

$$
2 V_{y}^{(t)}(x, y)-V_{y}^{(t)}(x, y-\varepsilon)-V_{y}^{(t)}(x, y+\varepsilon)=\frac{1}{\pi} \int_{-\infty}^{\infty} \frac{f_{y, \varepsilon}\left(\frac{2}{\pi} \log |s|\right) \cos \left(\frac{\pi}{2} x\right)}{\left(s-\sin \left(\frac{\pi}{2} x\right)\right)^{2}+\cos ^{2}\left(\frac{\pi}{2} x\right)} \mathrm{d} s=I,
$$

where

$$
\begin{aligned}
f_{y, \varepsilon}(h)= & 2\left(\Phi^{(t)}\right)^{\prime}(|y+h|) \operatorname{sgn}(y+h) \\
& -\left(\Phi^{(t)}\right)^{\prime}(|y-\varepsilon+h|) \operatorname{sgn}(y-\varepsilon+h)-\left(\Phi^{(t)}\right)^{\prime}(|y+\varepsilon+h|) \operatorname{sgn}(y+\varepsilon+h) .
\end{aligned}
$$

The expression $I$, after splitting it into integrals over the nonpositive and nonnegative halfline, and substitution $s= \pm e^{r}$, can be rewritten in the form

$$
I=\frac{1}{\pi} \int_{-\infty}^{\infty} f_{y, \varepsilon}\left(\frac{2}{\pi} r\right) g^{x}(r) \mathrm{d} r
$$


where

$$
g^{x}(r)=\frac{\cos \left(\frac{\pi}{2} x\right) e^{r}}{\left(e^{r}-\sin \left(\frac{\pi}{2} x\right)\right)^{2}+\cos ^{2}\left(\frac{\pi}{2} x\right)}+\frac{\cos \left(\frac{\pi}{2} x\right) e^{r}}{\left(e^{r}+\sin \left(\frac{\pi}{2} x\right)\right)^{2}+\cos ^{2}\left(\frac{\pi}{2} x\right)} .
$$

Observe that $f_{y, \varepsilon}(h) \leq 0$ for $h \geq-y$ and that we have $f_{y, \varepsilon}(-y+h)=-f_{y, \varepsilon}(-y-h)$ for all $h$. Furthermore, $g^{x}$ is even and, for $r>0$,

$$
\left(g^{x}\right)^{\prime}(r)=\frac{\cos \left(\frac{\pi}{2} x\right) e^{r}\left(1-e^{r}\right)}{\left[\left(e^{r}-\sin \left(\frac{\pi}{2} x\right)\right)^{2}+\cos ^{2}\left(\frac{\pi}{2} x\right)\right]^{2}}+\frac{\cos \left(\frac{\pi}{2} x\right) e^{r}\left(1-e^{r}\right)}{\left[\left(e^{r}+\sin \left(\frac{\pi}{2} x\right)\right)^{2}+\cos ^{2}\left(\frac{\pi}{2} x\right)\right]^{2}} \leq 0 .
$$

This implies $I \leq 0$ and, since $\varepsilon \in(0, x)$ was arbitrary, the function $y \mapsto V_{y}^{(t)}(x, y)$ is convex on $(0, \infty)$.

(iii) First note that

$$
V_{x y}^{(t)} \leq 0 \quad \text { for } x \in[0,1), y \geq 0 .
$$

Indeed, by (11), we have $V_{x}^{(t)}(0, y)=0$ for any $y \in \mathbb{R}$; this implies $V_{x y}^{(t)}(0, y)=0$ for all $y$. Furthermore, by (ii) and the fact that $V^{(t)}$ is harmonic, we have $V_{x y x}^{(t)}=-V_{y y y}^{(t)} \leq 0$ on $[0,1) \times[0, \infty)$ and hence (12) follows. Next, fix $y \geq 0$ and let $F(x)=y V_{x}^{(t)}(x, y)+$ $x V_{y}^{(t)}(x, y), x \in[0,1)$. Since $F(0)=0$, we will be done if we show that $F$ is nonincreasing. Using the harmonicity of $V^{(t)}$, we get

$$
\begin{aligned}
F^{\prime}(x) & =y V_{x x}^{(t)}(x, y)+V_{y}^{(t)}(x, y)+x V_{x y}^{(t)}(x, y) \\
& =\left(-y V_{y y}^{(t)}(x, y)+V_{y}^{(t)}(x, y)\right)+x V_{x y}^{(t)}(x, y) \leq 0,
\end{aligned}
$$

in virtue of (12) and (ii).

(iv) By (11), the partial derivatives of $V^{(t)}$ of odd order vanish at $(0,0)$ and hence so do those of $\operatorname{Im} G^{(t)}$, by Cauchy-Riemann equations. This implies $\left(G^{(t)}\right)^{(2 n+1)}(0)=0$ and the claim follows.

Consider the region $D=\left\{z \in \mathbb{C}:\left|2 \operatorname{Re} z^{1 / 2}\right| \leq 1\right\}$.

Lemma 2.3 The function $z \mapsto V^{(t)}\left(2 z^{1 / 2}\right), z \in D$, is harmonic.

Proof First notice that the function is well defined: in view of (11) it does not matter which square root of $z$ we take. The assertion is an immediate consequence of Lemma 2.2(iv): the function $z \mapsto G^{(t)}\left(2 z^{1 / 2}\right)$ is holomorphic and hence its real part is harmonic.

For a given $t \geq 0$, let $W^{(t)}:\left\{(w, z) \in \ell_{\mathbb{C}}^{2} \times \ell_{\mathbb{C}}^{2}:|w+\bar{z}| \leq 1\right\} \rightarrow \mathbb{R}$ be defined by the formula $W^{(t)}(w, z)=V^{(t)}\left(2(\langle w, \bar{z}\rangle)^{1 / 2}\right)$. The definition makes sense, in view of the following simple fact.

Lemma 2.4 For any $w, z \in \ell_{\mathbb{C}}^{2}$ we have

$$
2\left|\operatorname{Re}(\langle w, \bar{z}\rangle)^{1 / 2}\right| \leq|w+\bar{z}| \text { and } 2\left|\operatorname{Im}(\langle w, \bar{z}\rangle)^{1 / 2}\right| \leq|w-\bar{z}|
$$


Proof It suffices to establish the first estimate; the second follows by the substitution $-z$ in the place of $z$. We have

$$
\begin{aligned}
|w+\bar{z}|^{2} & =(w+\bar{z}) \cdot(w+\bar{z}) \\
& =|w|^{2}+|z|^{2}+2 \operatorname{Re}(\langle w, \bar{z}\rangle) \\
& \geq 2|w \cdot z|+2 \operatorname{Re}(\langle w, \bar{z}\rangle) \\
& =\left[(\langle w, \bar{z}\rangle)^{1 / 2}+\overline{(\langle w, \bar{z}\rangle)^{1 / 2}}\right]^{2} \\
& =\left(2 \operatorname{Re}(\langle w, \bar{z}\rangle)^{1 / 2}\right)^{2} .
\end{aligned}
$$

The proof is complete.

Lemma 2.5 We have $V^{(t)}(x, y) \geq \Phi^{(t)}(|y|)-C\left(\Phi^{(t)}\right)|x|$ on $\bar{S}$.

Proof We have decided to split the reasoning into two parts.

Step 1. We will show the pointwise estimate

$$
\Phi^{(t)}(|a+b|)+\Phi^{(t)}(|a-b|) \geq 2 \Phi^{(t)}(|a|)+2 \Phi^{(t)}(|b|)
$$

for any $t \geq 0$ and any $a, b \in \mathbb{R}$. By symmetry, we may assume that $|a| \geq|b|$ and, replacing $a, b$ by $-a$ and $-b$ if necessary, we may restrict ourselves to nonnegative $a$ and $b$. We consider four cases. Suppose first that $a-b \geq t$ and $b \geq t$. Then the inequality takes the form

$$
(a+b-t)^{2}+(a-b-t)^{2} \geq 2(a-t)^{2}+2(b-t)^{2},
$$

or $2 b \geq t$. This is clearly true, because of the assumption on $b$ we have just imposed. If $a-b \geq t$, but $b<t$, then (14) is equivalent to $2 b^{2} \geq 0$. Next, suppose that $a-b<t$ and $b \geq t$. Then (14) can be rewritten in the form $2 a t+2 b t \geq 3 t^{2}+(a-b)^{2}$. But

$$
2(a+b) t \geq 4 b t \geq 4 t^{2}>3 t^{2}+(a-b)^{2},
$$

as desired. Finally, assume that both $a-b$ and $b$ are smaller than $t$. If $a<t$, then the right-hand side of (14) vanishes and there is nothing to prove; otherwise, the estimate takes the form $(a+b-t)^{2} \geq 2(a-t)^{2}$. But this is evident: we have $a-b<t$, so $b>a-t$ and $a+b-t \geq 2(a-t)$.

Step 2. We turn to the assertion of the lemma. By (11), it suffices to establish the majorization for $x \in[0,1]$. In fact, we will be done if we do this for $x \in\{0,1\}$ (the left-hand side is a concave function of $x$, while the right-hand side is linear in $x$ ). If $x=1$, then both sides are equal. To deal with the case $x=0$, we apply (14) to get

$$
\Phi^{(t)}\left(\left|\frac{2}{\pi} \log \right| s|+y|\right)+\Phi^{(t)}\left(\left|\frac{2}{\pi} \log \right| s|-y|\right) \geq 2 \Phi^{(t)}\left(\left|\frac{2}{\pi} \log \right| s||\right)+2 \Phi^{(t)}(|y|) .
$$

Multiply both sides by $\left(\pi\left(s^{2}+1\right)\right)^{-1}$ and integrate over $\mathbb{R}$ with respect to the variable $s$ to obtain

$\left[V^{(t)}(0, y)+C\left(\Phi^{(t)}\right)\right]+\left[V^{(t)}(0,-y)+C\left(\Phi^{(t)}\right)\right] \geq 2\left[V^{(t)}(0,0)+C\left(\Phi^{(t)}\right)\right]+2 \Phi^{(t)}(|y|)$.

Combining this with $(11)$ and the equality $V^{(t)}(0,0)=0$, we get the desired majorization on the $y$-axis.

Lemma 2.6 For any $w, z \in \ell_{\mathbb{C}}^{2}$ such that $|w+\bar{z}| \leq 1$, we have

$$
W^{(t)}(w, z) \geq \Phi^{(t)}(|w-\bar{z}|)-C\left(\Phi^{(t)}\right)|w+\bar{z}| .
$$


Proof Fix $s \in \mathbb{R}$ and consider the function $F_{S}(x)=V^{(t)}\left(\sqrt{x^{2}+s}, x\right)$, defined for nonnegative $x$ satisfying $x^{2}+s \geq 0$. By Lemma 2.2(iii), this function is nonincreasing: indeed, we have

$$
F_{s}^{\prime}(x)=\frac{x}{\sqrt{x^{2}+s}} V_{x}^{(t)}\left(\sqrt{x^{2}+s}, x\right)+V_{y}^{(t)}\left(\sqrt{x^{2}+s}, x\right) \leq 0 .
$$

Therefore, by the previous lemma and (13),

$$
\begin{aligned}
& \Phi^{(t)}(|w-\bar{z}| / K)-C\left(\Phi^{(t)}\right)|w+\bar{z}| \\
& \quad \leq V^{(t)}(|w+\bar{z}|,|w-\bar{z}|)=F_{|w+\bar{z}|^{2}-|w-\bar{z}|^{2}}(|w-\bar{z}|) \\
& \quad \leq F_{|w+\bar{z}|^{2}-|w-\bar{z}|^{2}}\left(2\left|\operatorname{Im}(\langle w, \bar{z}\rangle)^{1 / 2}\right|\right)=V^{(t)}\left(2(\langle w, \bar{z}\rangle)^{1 / 2}\right),
\end{aligned}
$$

where the latter follows from the definition of $F_{|w+\bar{z}|^{2}-|w-\bar{z}|^{2}}$ and the identity

$$
\left(2\left|\operatorname{Re}(\langle w, \bar{z}\rangle)^{1 / 2}\right|\right)^{2}+|w-\bar{z}|^{2}-|w+\bar{z}|^{2}=\left(2\left|\operatorname{Im}(\langle w, \bar{z}\rangle)^{1 / 2}\right|\right)^{2} .
$$

We are ready to establish the $\Phi$-inequality for the Hilbert transform. First we prove it for the special functions $\Phi^{(t)}$.

Theorem 2.7 For any $t \geq 0$ and any function $g$ on $\mathbb{R}$ taking values in the unit ball of $\ell_{\mathbb{C}}^{2}$, we have

$$
\int_{\mathbb{R}} \Phi^{(t)}\left(\left|\mathcal{H}^{\mathbb{R}} g(x)\right|\right) \mathrm{d} x \leq C\left(\Phi^{(t)}\right)\|g\|_{L^{1}\left(\mathbb{R}, \ell_{\mathbb{C}}^{2}\right)}
$$

Proof We begin by showing an analogous statement in the periodic setting. Let $f$ be a Borel function on $\mathbb{T}$ taking values in the unit ball of $\ell_{\mathbb{C}}^{2}$ and let $f_{+}, f_{-}$denote the harmonic extensions of $P_{+}^{\mathbb{T}} f-\frac{1}{2} \hat{f}(0)$ and $\overline{P_{-}^{\mathbb{T}} f}+\frac{1}{2} \bar{f}(0)$ to the unit disc $\mathbb{D}$. By Theorem 2.1, the function $W^{(t)}$ is pluriharmonic and thus $W^{(t)}\left(f_{+}, f_{-}\right)$is harmonic on $\mathbb{D}$ (note that $|f|=$ $\left|f_{+}+\overline{f_{-}}\right| \leq 1$ guarantees that $W^{(t)}\left(f_{+}, f_{-}\right)$is well defined). Apply the mean-value property and Lemma 2.2(i) to get

$$
\frac{1}{2 \pi} \int_{\mathbb{T}} W^{(t)}\left(f_{+}(\zeta), f_{-}(\zeta)\right) \mathrm{d} \zeta=W^{(t)}\left(\frac{1}{2} \hat{f}(0), \frac{1}{2} \hat{\hat{f}(0)}\right)=V^{(t)}(|\hat{f}(0)|, 0) \leq 0 .
$$

Combine this with (15) to obtain

$$
\frac{1}{2 \pi} \int_{\mathbb{T}} \Phi^{(t)}\left(\left|\mathcal{H}^{\mathbb{T}} f(\zeta)\right|\right) \mathrm{d} \zeta \leq C\left(\Phi^{(t)}\right)\|f\|_{L^{1}\left(\mathbb{T}, \ell_{\mathbb{C}}^{2}\right.} .
$$

Let us turn to (16), the nonperiodic version of the above estimate. Pick an $\ell_{\mathbb{C}}^{2}$-valued function $g=\left(g^{1}, g^{2}, \ldots\right)$ on the line and let $u^{j}=\operatorname{Re} g^{j}, v^{j}=\operatorname{Im} g^{j}, j=1,2, \ldots$ Introduce the functions $h_{n}=\left(h_{n}^{1}, h_{n}^{2}, \ldots\right), k_{n}=\left(k_{n}^{1}, k_{n}^{2}, \ldots\right)$ by

$$
\begin{aligned}
& h_{n}^{j}(x)=\frac{1}{2 \pi n} \int_{-\pi n}^{\pi n} u^{j}(t) \cot \frac{x-t}{2 n} \mathrm{~d} t, \\
& k_{n}^{j}(x)=\frac{1}{2 \pi n} \int_{-\pi n}^{\pi n} v^{j}(t) \cot \frac{x-t}{2 n} \mathrm{~d} t,
\end{aligned}
$$


for $j, n \geq 1$. As shown by Zygmund [22], for any fixed $j$ we have $h_{n}^{j} \rightarrow \mathcal{H}^{\mathbb{R}} u^{j}$ and $k_{n}^{j} \rightarrow \mathcal{H}^{\mathbb{R}} v^{j}$ a.e. as $n \rightarrow \infty$. On the other hand, the function $x \mapsto h_{n}^{j}(n x)+i k_{n}^{j}(n x)$ is precisely the periodic Hilbert transform of the function $x \mapsto g^{j}(n x),|x| \leq \pi$ [see (1)]. Therefore, by (17),

$$
\begin{aligned}
\int_{-n \pi}^{n \pi} \Phi^{(t)}\left(\left|h_{n}(x)+i k_{n}(x)\right|\right) \mathrm{d} x & =n \int_{-\pi}^{\pi} \Phi^{(t)}\left(\mathcal{H}^{\mathbb{T}} g(n x)\right) \mathrm{d} x \\
& \leq n C\left(\Phi^{(t)}\right) \int_{-\pi}^{\pi}|g(n x)| \mathrm{d} x \\
& =C\left(\Phi^{(t)}\right) \int_{-\pi n}^{\pi n}|g(x)| \mathrm{d} x .
\end{aligned}
$$

It remains to let $n \rightarrow \infty$ to obtain (16), in view of Lebesgue's monotone convergence theorem.

Now we extend the estimate (16) to the class $\mathcal{C}$ (see Sect. 1). Namely, we will prove the following fact.

Theorem 2.8 Assume that $\Phi \in \mathcal{C}$. Then for any function $g$ on $\mathbb{R}$ taking values in the unit ball of $\ell_{\mathbb{C}}^{2}$, we have

$$
\int_{\mathbb{R}} \Phi\left(\left|\mathcal{H}^{\mathbb{R}} g(x)\right|\right) \mathrm{d} x \leq C(\Phi)\|g\|_{L^{1}\left(\mathbb{R}, \ell_{\mathbb{C}}^{2}\right)}
$$

Proof Let $\mu$ be the unique nonnegative measure on $\mathbb{R}_{+}$satisfying $\mu((a, b])=\Phi_{+}^{\prime \prime}(b)$ $-\Phi_{+}^{\prime \prime}(a)$ for all $0 \leq a<b$. Here $\Phi_{+}^{\prime \prime}$ stands for the right-hand derivative of the convex function $\Phi^{\prime}$. Integrating by parts, we get the identity

$$
\Phi(t)=\frac{\Phi_{+}^{\prime \prime}(0) t^{2}}{2}+\frac{1}{2} \int_{0}^{t}(s-t)^{2} \mathrm{~d} \mu(s)=\frac{\Phi_{+}^{\prime \prime}(0)}{2} \Phi^{(0)}(t)+\frac{1}{2} \int_{0}^{\infty} \Phi^{(s)}(t) \mathrm{d} \mu(s) .
$$

Thus the claim follows from (16) and Fubini's theorem:

$$
\begin{aligned}
& \int_{\mathbb{R}} \Phi\left(\left|\mathcal{H}^{\mathbb{R}} g(x)\right|\right) \mathrm{d} x \\
& =\frac{\Phi_{+}^{\prime \prime}(0)}{2} \int_{\mathbb{R}} \Phi^{(0)}\left(\left|\mathcal{H}^{\mathbb{R}} g(x)\right|\right) \mathrm{d} x+\frac{1}{2} \int_{0}^{\infty} \int_{\mathbb{R}} \Phi^{(s)}\left(\left|\mathcal{H}^{\mathbb{R}} g(x)\right|\right) \mathrm{d} x \mathrm{~d} \mu(s) \\
& \leq \frac{\Phi^{\prime \prime}(0+)}{2} C\left(\Phi^{0}\right)+\frac{1}{2} \int_{0}^{\infty} C\left(\Phi^{(s)}\right) \mathrm{d} \mu(s)=C(\Phi) .
\end{aligned}
$$

The proof is finished.

We conclude this section by saying that both (16) and (18) are sharp. This will be shown in Sect. 4 below. 


\section{Estimates for the truncated Hilbert transform and the segment multiplier}

In this section we will show how to deduce the inequalities (4) and (6) from the results presented in Sect. 2. In fact, we will first establish appropriate versions of (18) for $\mathcal{H}_{r}$ and $\mathcal{S}_{[a, b]}$, and then proceed using duality arguments. We start from several simple observations. First, assume that $f$ is a function on $\mathbb{R}$ taking values in $\ell_{\mathbb{C}}^{2}$ and, for a given $s \in \mathbb{R}$, define the linear operator $M_{S}$ by

$$
M_{S} f(x)=e^{2 \pi i s x} f(x), \quad x \in \mathbb{R} .
$$

Then it can be easily computed that the Fourier transform of this object equals

$$
\widehat{M_{s} f}(\xi)=\int_{\mathbb{R}} f(x) e^{2 \pi i s x} e^{-2 \pi i \xi x} \mathrm{~d} x=\hat{f}(\xi-s)
$$

and therefore we have the identity

$$
M_{s} \widehat{\mathcal{H}}^{\mathbb{R}} M_{-s} f(\xi)=-i \operatorname{sgn}(\xi-s) \hat{f}(\xi) .
$$

Consequently,

$$
\begin{aligned}
\mathcal{H}_{r} f(x) & =-i \int_{\mathbb{R}}\left(-\chi_{(-\infty,-r)}(\xi)+\chi_{(r, \infty)}(\xi)\right) \hat{f}(\xi) e^{2 \pi i x \xi} \mathrm{d} \xi \\
& =-\frac{i}{2} \int_{\mathbb{R}}(\operatorname{sgn}(\xi-r)+\operatorname{sgn}(\xi+r)) \hat{f}(\xi) e^{2 \pi i x \xi} \mathrm{d} \xi \\
& =\frac{1}{2}\left(M_{r} \mathcal{H}^{\mathbb{R}} M_{-r}+M_{-r} \mathcal{H}^{\mathbb{R}} M_{r}\right) f(x) .
\end{aligned}
$$

Fix a function $f$ on $\mathbb{R}$ taking values in the unit ball of $\ell_{\mathbb{C}}^{2}$ and let $r$ be a given positive number. The functions $M_{r} f, M_{-r} f$ are also bounded by 1 and therefore, by Jensen inequality,

$$
\begin{aligned}
\int_{\mathbb{R}} \Phi\left(\left|\mathcal{H}_{r} f(x)\right|\right) \mathrm{d} x & \leq \frac{1}{2} \int_{\mathbb{R}} \Phi\left(\left|\left(M_{r} \mathcal{H}^{\mathbb{R}} M_{-r} f\right)(x)\right|\right) \mathrm{d} x+\frac{1}{2} \int_{\mathbb{R}} \Phi\left(\left|\left(M_{-r} \mathcal{H}^{\mathbb{R}} M_{r} f\right)(x)\right|\right) \mathrm{d} x \\
& =\frac{1}{2} \int_{\mathbb{R}} \Phi\left(\left|\left(\mathcal{H}^{\mathbb{R}} M_{r} f\right)(x)\right|\right) \mathrm{d} x+\frac{1}{2} \int_{\mathbb{R}} \Phi\left(\left|\left(\mathcal{H}^{\mathbb{R}} M_{-r} f\right)(x)\right|\right) \mathrm{d} x \\
& \leq C(\Phi) \frac{\left\|M_{-r} f\right\|_{L^{1}\left(\mathbb{R}, \ell_{\mathbb{C}}^{2}\right)}+\left\|M_{r} f\right\|_{L^{1}\left(\mathbb{R}, \ell_{\mathbb{C}}^{2}\right)}}{2} \\
& =C(\Phi)\|f\|_{L^{1}\left(\mathbb{R}, \ell_{\mathbb{C}}^{2}\right)}
\end{aligned}
$$

Next, we turn to the dual estimate. Let $\Psi=\Phi^{*}$ be a given element of $\mathcal{C}^{*}$. For any Borel $f: \mathbb{R} \rightarrow \ell_{\mathbb{C}}^{2}$ and any $A \subset \mathbb{R}$ with $|A|<\infty$, we write

$$
\int_{A}\left|\mathcal{H}_{r} f(x)\right| \mathrm{d} x=\int_{\mathbb{R}} \mathcal{H}_{r} f(x) \overline{g(x)} \mathrm{d} x,
$$

where $g(x)=\chi_{A} \mathcal{H}_{r} f(x) /\left|\mathcal{H}_{r} f(x)\right|$ (if $\mathcal{H}_{r} f(x)=0$, we put $g(x)=0$ ). We have also used the more convenient notation $a b=\langle a, \bar{b}\rangle$ for $a, b \in \ell_{\mathbb{C}}^{2}$. Consequently, by Parseval's 
relation and Young's inequality (i.e., the bound $s t \leq \Phi(t)+\Psi(s)$ for nonnegative $s, t$ : see the definition of $\mathcal{C}^{*}$ in the introductory section),

$$
\begin{aligned}
& \int_{\mathbb{R}} \mathcal{H}_{r} f(x) \overline{g(x)} \mathrm{d} x=\int_{\mathbb{R}} \widehat{\mathcal{H}_{r} f}(\xi) \overline{\hat{g}(\xi)} \mathrm{d} \xi \\
& =-i \int_{\mathbb{R}} \hat{f}(\xi) \overline{\left(-\chi_{(-\infty,-r)}(\xi)+\chi_{(r, \infty)}(\xi)\right) \hat{g}(\xi)} \mathrm{d} \xi \\
& =-\int_{\mathbb{R}} \hat{f}(\xi) \overline{\mathcal{H}_{r} g}(\xi) \mathrm{d} \xi \\
& =-\int_{\mathbb{R}} f(x) \overline{\mathcal{H}_{r} g(x)} \mathrm{d} x \\
& \leq \int_{\mathbb{R}} \Psi(|f(x)|) \mathrm{d} x+\int_{\mathbb{R}} \Phi\left(\left|\mathcal{H}_{r} g(x)\right|\right) \mathrm{d} x \\
& \leq \int_{\mathbb{R}} \Psi(|f(x)|) \mathrm{d} x+C(\Phi)\|g\|_{L^{1}\left(\mathbb{R}, \ell_{\mathbb{C}}^{2}\right.} .
\end{aligned}
$$

It remains to observe that the $L^{1}$-norm of $g$ does not exceed the measure of $A$. The reasoning leading to the estimate (6) for the segment multiplier $S$ is similar. Fix an interval $[a, b]$ and note that

$$
\begin{aligned}
\mathcal{S}_{[a, b]} f(x) & =\int_{a}^{b} \hat{f}(\xi) e^{2 \pi i x \xi} \mathrm{d} \xi \\
& =e^{-2 \pi i x s} \int_{a+s}^{b+s} \hat{f}(\xi-s) e^{2 \pi i x \xi} \mathrm{d} \xi \\
& =e^{-2 \pi i x s} S_{[a+s, b+s]} M_{s} f(x) .
\end{aligned}
$$

Taking $s=-(a+b) / 2$, we see that the line segment $[a+s, b+s]=[(a-b) / 2,(b-a) / 2]$ is symmetric about 0 . Such symmetric multipliers admit the following convenient form: for any $r>0$ and any $f$ we have

$$
\begin{aligned}
S_{[-r, r]} f(x) & =\int_{\mathbb{R}} \hat{f}(\xi) \chi_{[-r, r]}(\xi) e^{2 \pi i x \xi} \mathrm{d} \xi \\
& =\frac{1}{2 \pi} \int_{\mathbb{R}} f(t) \frac{e^{2 \pi i r(x-t)}-e^{-2 \pi i r(x-t)}}{x-t} \mathrm{~d} t \\
& =\frac{1}{2}\left(M_{r} \mathcal{H}^{\mathbb{R}} M_{-r} f\right)(x)-\frac{1}{2}\left(M_{-r} \mathcal{H}^{\mathbb{R}} M_{r} f\right)(x) .
\end{aligned}
$$

The two facts above allow us to show an appropriate version of (18) for the segment multiplier. Let $f$ be a given function on the real line, taking values in the unit ball of $\ell_{\mathbb{C}}^{2}$, and let $[a, b]$ be a fixed subinterval of $\mathbb{R}$. For any $s \in \mathbb{R}$, the function $M_{s} f$ is also bounded by 1 and therefore, 
by Jensen inequality,

$$
\begin{aligned}
\int_{\mathbb{R}} \Phi\left(\left|\mathcal{S}_{[a, b]} f(x)\right|\right) \mathrm{d} x= & \int_{\mathbb{R}} \Phi\left(\left|\left(S_{[(a-b) / 2,(b-a) / 2]} M_{-(a+b) / 2} f\right)(x)\right|\right) \mathrm{d} x \\
\leq & \frac{1}{2} \int_{\mathbb{R}} \Phi\left(\left|\left(M_{(b-a) / 2} \mathcal{H}^{\mathbb{R}} M_{(a-b) / 2} M_{-(a+b) / 2} f\right)(x)\right|\right) \mathrm{d} x \\
& +\frac{1}{2} \int_{\mathbb{R}} \Phi\left(\left|\left(M_{(a-b) / 2} \mathcal{H}^{\mathbb{R}} M_{(b-a) / 2} M_{-(a+b) / 2} f\right)(x)\right|\right) \mathrm{d} x \\
= & \left.\left.\frac{1}{2} \int_{\mathbb{R}} \Phi\left(\mid \mathcal{H}^{\mathbb{R}} M_{-b} f\right)(x) \mid\right) \mathrm{d} x+\frac{1}{2} \int_{\mathbb{R}} \Phi\left(\mid \mathcal{H}^{\mathbb{R}} M_{-a} f\right)(x) \mid\right) \mathrm{d} x \\
\leq & C(\Phi) \frac{\left\|M_{-b} f\right\|_{L^{1}\left(\mathbb{R}, \ell_{\mathbb{C}}^{2}\right)}+\left\|M_{-a} f\right\|_{L^{1}\left(\mathbb{R}, \ell_{\mathbb{C}}^{2}\right)}}{2} \\
= & C(\Phi)\|f\|_{L^{1}\left(\mathbb{R}, \ell_{\mathbb{C}}^{2}\right)}
\end{aligned}
$$

Next, we turn to (6). For any Borel $f: \mathbb{R} \rightarrow \ell_{\mathbb{C}}^{2}$ and any $A \subset \mathbb{R}$ with $|A|<\infty$, we write

$$
\int_{A}\left|\mathcal{S}_{[a, b]} f(x)\right| \mathrm{d} x=\int_{\mathbb{R}} \mathcal{S}_{[a, b]} f(x) \overline{g(x)} \mathrm{d} x,
$$

where $g(x)=\chi_{A} \mathcal{S}_{[a, b]} f(x) /\left|\mathcal{S}_{[a, b]} f(x)\right|$ (if $\mathcal{S}_{[a, b]} f(x)=0$, we put $g(x)=0$ ). An application of Parseval's relation and Young's inequality yields

$$
\begin{aligned}
\int_{\mathbb{R}} \mathcal{S}_{[a, b]} f(x) \overline{g(x)} \mathrm{d} x & =\int_{\mathbb{R}} \widehat{\mathcal{S}_{[a, b]} f(\xi) \overline{\hat{g}(\xi)} \mathrm{d} \xi} \\
& =\int_{\mathbb{R}} \hat{f}(\xi) \overline{\chi_{[a, b]}(\xi) \hat{g}(\xi)} \mathrm{d} \xi \\
& =\int_{\mathbb{R}} \hat{f}(\xi) \overline{\widehat{\mathcal{S}_{[a, b]}}}(\xi) \mathrm{d} \xi \\
& =\int_{\mathbb{R}} f(x) \overline{\mathcal{S}_{[a, b]} g(x)} \mathrm{d} x \\
& \leq \int_{\mathbb{R}} \Psi(|f(x)|) \mathrm{d} x+\int_{\mathbb{R}} \Phi\left(\left|\mathcal{S}_{[a, b]} g(x)\right|\right) \mathrm{d} x \\
& \leq \int_{\mathbb{R}} \Psi(|f(x)|) \mathrm{d} x+C(\Phi)|| g \|_{L^{1}\left(\mathbb{R}, \ell_{\mathbb{C}}^{2}\right)}
\end{aligned}
$$

Since the $L^{1}$-norm of $g$ does not exceed the measure of $A$, the proof is complete.

\section{Sharpness of (4) and a lower bound related to (6)}

For the sake of convenience and clarity of the exposition, we have decided to split this section into three parts. 
Sharpness of (16) and (18). We will exploit Davis' argument from [2]. Let $\mathbb{D}$ be the unit disc of $\mathbb{C}$ and let $H$ denote the upper halfplane. Consider a function $K(z)=(1+z)^{2} / 4 z$, which maps the half disc $\mathbb{D} \cap H$ onto $H$, and the boundary of $\mathbb{D} \cap H$ onto $\mathbb{R}$. Let $L$ be the inverse of $K$. Then $L$ maps $[0,1]$ onto the half circle $\left\{e^{i \theta}: 0 \leq \theta \leq \pi\right\}$, and $\mathbb{R} \backslash[0,1]$ onto $(-1,1)$ :

$$
L(x)= \begin{cases}1-2 x-2 \sqrt{x^{2}-x} & \text { if } x<0, \\ 1-2 x+2 \sqrt{x^{2}-x} & \text { if } x>1 .\end{cases}
$$

Let $d_{n}$ be the density of $L^{n}([0,1])$ on $\mathbb{T}$ with respect to the normalized Lebesgue's measure, i.e. for any $-\pi<\alpha<\beta<\pi$,

$$
\int_{\alpha}^{\beta} d_{n}\left(e^{i \theta}\right) \frac{\mathrm{d} \theta}{2 \pi}=\left|\left\{r \in[0,1]: L_{n}(r) \in\left\{e^{i \theta}: \alpha<\theta<\beta\right\}\right\}\right| .
$$

Then (see Lemma 3 in [2]) $d_{n} \rightarrow 1$ uniformly on $\mathbb{T}$. Next we introduce a conformal mapping $F$ of $\mathbb{D}$ onto the strip $\{z:|\operatorname{Re} z| \leq 1\}$ by

$$
F(z)=(2 i / \pi) \log [(i z-1) /(z-i)]-1 .
$$

For a fixed integer $n$, define $f_{n}: \mathbb{R} \rightarrow[-1,1]$ by the formula $f_{n}(x)=\operatorname{Re}\left(F\left(L^{n}(x)\right)\right)$. By (21) and the equality $F(0)=0$, the function $f$ converges rapidly to 0 on each set of the form $\mathbb{R} \backslash[-\varepsilon, 1+\varepsilon], \varepsilon>0$, and consequently

$$
\limsup _{n \rightarrow \infty}\left\|f_{n}\right\|_{L^{1}(\mathbb{R}, \mathbb{R})} \leq 1 .
$$

The mapping $z \mapsto F\left(L^{n}(z)\right)$ is conformal and satisfies $\lim _{z \rightarrow \infty} F\left(L^{n}(z)\right)=0$, so $\mathcal{H}^{\mathbb{R}} f_{n}=$ $\operatorname{Im} F\left(L^{n}(\cdot)\right)$. Therefore,

$$
\begin{aligned}
\int_{\mathbb{R}} \Phi\left(\left|\mathcal{H}^{\mathbb{R}} f_{n}(x)\right|\right) \mathrm{d} x & =\int_{\mathbb{R}} \Phi\left(\left|\operatorname{Im} F\left(L^{n}(x)\right)\right|\right) \mathrm{d} x \\
& \geq \int_{0}^{1} \Phi\left(\left|\operatorname{Im} F\left(L^{n}(x)\right)\right|\right) \mathrm{d} x \\
& =\int_{-\pi}^{\pi} \Phi\left(\left|\operatorname{Im} F\left(e^{i \theta}\right)\right|\right) d_{n}\left(e^{i \theta}\right) \frac{\mathrm{d} \theta}{2 \pi} \\
& \rightarrow \int_{-\pi}^{\pi} \Phi\left(\left|\operatorname{Im} F\left(e^{i \theta}\right)\right|\right) \frac{\mathrm{d} \theta}{2 \pi}=C(\Phi),
\end{aligned}
$$

The latter equality follows from the identity $\operatorname{Im} F\left(e^{i \theta}\right)=(2 / \pi) \log |\sin \theta /(1-\cos \theta)|$ and the substitution $t=\sin \theta /(1-\cos \theta)$ under the integral.

Sharpness of (4). Let $r_{0}$ be a fixed positive number and let $\Psi$ be a given element of the class $\mathcal{C}^{*}$. Assume that the inequality (4) holds with a certain constant $C$. For any $s>0$ and any $f$ we have $\mathcal{H}_{r} f=D_{s} \mathcal{H}_{r / s} D_{1 / s} f$, where $D_{s}$ stands for the dilation operator: $D_{s} f(x)=f(s x)$ 
for all $x \in \mathbb{R}$. This identity implies that (4) holds with the constant $C$ for all $r$ : indeed,

$$
\begin{aligned}
\int_{A}\left|\mathcal{H}_{r} f(x)\right| \mathrm{d} x & =\int_{A}\left|D_{r / r_{0}} \mathcal{H}_{r_{0}} D_{r_{0} / r} f(x)\right| \mathrm{d} x \\
& =\frac{r_{0}}{r} \int_{r A / r_{0}}\left|\mathcal{H}_{r_{0}} D_{r_{0} / r} f(x)\right| \mathrm{d} x \\
& \leq \frac{r_{0}}{r} \int_{\mathbb{R}} \Psi\left(\left|D_{r_{0} / r} f(x)\right|\right) \mathrm{d} x+C \cdot \frac{r_{0}}{r}\left|r A / r_{0}\right| \\
& =\int_{\mathbb{R}} \Psi(|f(x)|) \mathrm{d} x+C \cdot|A| .
\end{aligned}
$$

On the other hand, suppose that $f: \mathbb{R} \rightarrow \mathbb{R}$ is a square-integrable function. By Plancherel's theorem and Lebesgue's dominated convergence theorem, we have

$$
\lim _{r \rightarrow 0} \widehat{\mathcal{H}_{r} f}=\widehat{\mathcal{H}^{\mathbb{R}} f}
$$

in $L^{2}(\mathbb{R})$. Therefore, there is a sequence $\left(r_{n}\right)_{n \geq 1}$ decreasing to 0 such that $\mathcal{H}_{r_{n}} f \rightarrow \mathcal{H}^{\mathbb{R}} f$ almost everywhere and thus, by Fatou's lemma and (23), for any real-valued function $f \in$ $L^{2}(\mathbb{R})$

$$
\int_{A}\left|\mathcal{H}^{\mathbb{R}} f(x)\right| \mathrm{d} x \leq \int_{\mathbb{R}} \Psi(|f(x)|) \mathrm{d} x+C \cdot|A| .
$$

By a standard approximation, we obtain that this inequality holds for all real-valued integrable $f$. Therefore, $C$ cannot be smaller than the optimal constant for the Hilbert transform, which is precisely $C(\Phi)$, in view of the reasoning presented above. Consequently, $C(\Phi)$ is indeed the best in (4).

Lower bound related to (6). We turn to the analysis of the segment multiplier $\mathcal{S}_{[a, b]}$. Let $\Psi$ be a fixed element of the class $\mathcal{C}^{*}$ and let $C$ be the best constant in (6). In view of (20), this constant does not change if we replace $[a, b]$ by any interval of the same length. Furthermore, we easily check that for any $s>0$ we have $D_{s} \mathcal{S}_{[a, b]} D_{1 / s}=\mathcal{S}_{[s a, s b]}$ which, by the same reasoning as above, gives that the best constants for $\mathcal{S}_{[a, b]}$ and $\mathcal{S}_{[s a, s b]}$ coincide. In conclusion, all the multipliers corresponding to segments of finite length satisfy (6) with the constant $C$. Repeating the above argument using Plancherel's theorem and Fatou's lemma, we get the analogues of (6) for $P_{ \pm}^{\mathbb{R}}$ and therefore,

$$
\int_{A}\left|\mathcal{H}^{\mathbb{R}} f(x)\right| \mathrm{d} x=\int_{A}\left|P_{+}^{\mathbb{R}} f(x)-P_{-}^{\mathbb{R}} f(x)\right| \mathrm{d} x \leq 2 \int_{\mathbb{R}} \Psi(|f(x)|) \mathrm{d} x+2 C \cdot|A| .
$$

This gives the lower bound $C \geq C\left((2 \Psi)^{*}\right) / 2$. To give a concrete example, consider the inequality

$$
\int_{A}\left|\mathcal{S}_{[a, b]} f(x)\right| \mathrm{d} x \leq K \int_{\mathbb{R}}[(|f(x)|+1) \log (|f(x)|+1)-|f(x)|] \mathrm{d} x+C \cdot|A|
$$

(this corresponds to the choice $\Psi(t)=K((t+1) \log (t+1)-t)$ : see example (ii) in Sect. 1). An analogous inequality holds for $\mathcal{H}^{\mathbb{R}}$ (with some finite $C$ ) if and only if $K>2 / \pi$. Thus, the above arguments imply that (24) does not hold with any finite $C$ when $K \leq 1 / \pi$. 
Acknowledgments The author would like to thank an anonymous referee for the careful reading of the first version of the paper and for several helpful comments and suggestions. The research was partially supported by Polish Ministry of Science and Higher Education (MNiSW) grant IP2011 039571 'Iuventus Plus'.

Open Access This article is distributed under the terms of the Creative Commons Attribution License which permits any use, distribution, and reproduction in any medium, provided the original author(s) and the source are credited.

\section{References}

1. Bennett, C.: A best constant for Zygmund's conjugate function inequality. Proc. Am. Math. Soc. 56, 256-260 (1976)

2. Davis, B.: On the weak type $(1,1)$ inequality for conjugate functions. Proc. Am. Math. Soc. 44, 307-311 (1974)

3. de Carli, L., Laeng, E.: On the (p, p) norm of monotonic Fourier multipliers. C. R. Acad. Sci. Paris Sér. I Math. 330(8), 657-662 (2000)

4. de Carli, L., Laeng, E.: Sharp $L^{p}$ estimates for the segment multiplier. Collect. Math. 51(3), 309-326 (2000)

5. Essén, E.M.: A superharmonic proof of the M. Riesz conjugate function theorem. Ark. Mat. 22, 241-249 (1984)

6. Essén, M., Shea, D.F., Stanton, C.S.: Best constants in Zygmund's inequality for conjugate functions. In: Heinonen, Kilpeläinen, Koskela (eds.) A volume dedicated to Olli Martio on his 60th birthday. Report 83. Department of Mathematics, University of Jyväskila, pp. 73-80 (2001)

7. Essén, M., Shea, D.F., Stanton, C.S.: Sharp $L \log ^{\alpha} L$ inequalities for conjugate functions. Ann. Inst. Fourier Grenoble 52(2), 623-659 (2002)

8. Gamelin, T.W.: Uniform Algebras and Jensen measures. London Mathematical Society. Lecture Notes Series, vol. 32. Cambridge University Press, Cambridge (1978)

9. Gohberg, I., Krupnik, N.: Norm of the Hilbert transformation in the $L_{p}$ space. Funct. Anal. Pril. 2, 91-92 [in Russian; English translation in Funct. Anal. Appl. 2, 180-181 (1968)]

10. Grafakos, L.: Classical Fourier Analysis, 2nd edn. Graduate Texts in Mathematics, vol. 249. Springer, New York (2008)

11. Hollenbeck, B., Verbitsky, I.E.: Best constants for the Riesz projection. J. Funct. Anal. 175, 370-392 (2000)

12. Hollenbeck, B., Verbitsky, I.E.: Best constant inequalities involving the analytic and co-analytic projections. Oper. Theory Adv. Appl. 202, 285-296 (2010)

13. Laeng, E.: Sharp norm inequalities for the truncated Hilbert transform. J. Math. Inequal. 3(1), 123-127 (2009)

14. O'Neil, R., Weiss, G.: The Hilbert transform and rearrangement of functions. Studia Math. 23, 189-198 (1963)

15. Osȩkowski, A.: Logarithmic estimates for the Hilbert transform and the Riesz projection. Arch. Math. 98, 153-161 (2012)

16. Osękowski, A.: Sharp logarithmic inequalities for Riesz transforms. J. Funct. Anal. 263, 89-108 (2012)

17. Pełczyński, A.: Norms of classical operators in function spaces. In: Colloque en l'honneur de Laurent Schwartz, vol. 1. Asterisque 131, pp. 137-162 (1985)

18. Pichorides, S.K.: On the best values of the constants in the theorems of M. Riesz, Zygmund and Kolmogorov. Studia Math. 44, 165-179 (1972)

19. Riesz, M.: Sur les fonctions conjugées. Math. Z. 27, 218-244 (1927)

20. Range, R.M.: Holomorphic Functions and Integral Representations in Several Complex Variables. Graduate Texts in Mathematics, vol. 108. Springer, New York (1986)

21. Verbitsky, I.E.: Estimate of the norm of a function in a Hardy space in terms of the norms of its real and imaginary part. Mat. Issled. 54, 16-20 (1980). [in Russian; English translation in Am. Math. Soc. Transl. (2) 124, 11-15 (1984)]

22. Zygmund, A.: Sur les fonctions conjugées. Fund. Math. 13, 284-303 (1929) 